\title{
Alternating hemiplegia of childhood presenting as recurrent apnoea in a term newborn infant
}

\section{Dear Editor,}

Recurrent apnoea in a term infant is usually pathologic, warranting a thorough aetiologic evaluation. An accurate diagnosis is essential in guiding subsequent management and understanding long-term prognosis.

We report a full term (38 weeks) infant, with birth weight $3.1 \mathrm{~kg}$, born well via normal vaginal delivery. Antenatal history and scans were unremarkable. Parents were non-consanguineous with no family history of neurodevelopmental disorders. At 7 hours of life, she had a sudden episode of skin mottling, poor perfusion and lethargy. She was treated for presumed sepsis with intravenous ampicillin, cefotaxime and acyclovir, and given continuous positive airway pressure (cPAP). At 24 hours, a second episode involved apnoea, skin mottling, poor perfusion, gaze deviation and limb posturing. Pertinent examination findings were hypotonia, significant head lag and intermittent oscillatory conjugate eye movements. There was no dysmorphism, cranial nerve deficits or motor deficits. The Moro, deep tendon and grasp reflexes were symmetrical bilaterally.

Over subsequent weeks, she continued to have recurrent episodes of apnoea in wakefulness (never in sleep) up to 4 times/day, each lasting 15-60 seconds. Some events were associated with eye deviation, clonic jerking of the right side, and/or dystonic limb posturing, but unilateral weakness was not observed. Episodes were unprovoked and not related to feeding, pharyngeal suctioning or positional change, and persisted despite treatment with intravenous phenobarbitone (loading doses of $20 \mathrm{mg} / \mathrm{kg}$ and $10 \mathrm{mg} / \mathrm{kg}$ dose, and maintenance of $6 \mathrm{mg} / \mathrm{kg} /$ day) and oral levetiracetam (40mg/kg/day). Video electroencephalogram (EEG) demonstrated a normal neonatal background organisation and no epileptiform activity even during the typical events.

Given the clinical presentation, examination findings and investigation results, differential diagnosis considered include seizures, movement disorder, gastro-oesophageal reflux and sepsis. A normal brain magnetic resonance imaging (MRI) scan excluded intracranial structural pathologies. Infection screen was negative-sterile blood, urine and cerebrospinal fluid (CSF) cultures; negative viral polymerase chain reaction for respiratory viruses (nasal swabs), CSF herpes simplex virus and enterovirus; and negative toxoplasmosis and cytomegalovirus serology. An extensive metabolic screen was normal (plasma ammonia, uric acid, creatinine kinase, homocysteine, very long chain fatty acids, phytanic acid, pristanic acid, urine organic acid, and CSF:plasma ratios of lactate and glycine), excluding rarer metabolic causes.

Clinical exome analysis revealed a de novo heterozygous splice-site variant in the ATP1A3 gene (NM_001256214.2:c.2960+2delT), in keeping with a neonatal onset of alternating hemiplegia of childhood (AHC). Following this result, she was started on incremental doses of oral flunarizine $(5 \mathrm{mg}$ every other day to $3 \mathrm{mg}$ daily) following which the episodes of apnoea reduced in frequency. Intranasal midazolam $0.2 \mathrm{mg} / \mathrm{kg} / \mathrm{dose}$ was prescribed for prolonged episodes lasting $>5$ minutes. She was weaned from cPAP to home oxygen $(2 \mathrm{~L} / \mathrm{min})$ prior to discharge.

Discussion. Apnoea, the absence of respiratory effort for $>20$ seconds or shorter pauses associated bradycardia or desaturation, can be classified as central, obstructive or mixed. Common causes of apnoea in term infants, which is almost always pathological, ${ }^{1}$ is summarised in Table 1.

In the evaluation of these infants, a detailed maternal medical, medication, birth and family history, and physical examination should be followed by investigations to identify treatable aetiologies such as sepsis, pneumonia or meningitis and correctible metabolic derangements. An EEG and a 24-hour oesophageal $\mathrm{pH}$-impedance study will exclude seizures and gastro-oesophageal reflux. In infants with a sudden deterioration, apnoea and a bulging fontanelle, urgent neuroimaging and neurosurgical evaluation may be required for hydrocephalus or intracranial bleeding. A genetic consult will be necessary in infants with recurrent apnoea without an identifiable cause. An important differential is congenital central hypoventilation syndrome, caused by phenylalanine repeat expansions in the $\mathrm{PHOX} 2 \mathrm{~B}$ gene, with apnoea mainly when asleep.

AHC ( 1 in 1,000,000 births) is caused by an autosomal dominant mutation in the ATP1A3 gene which encodes the alpha-3 catalytic subunit of the $\mathrm{Na}^{+} / \mathrm{K}^{+}$ATPase transmembrane ion pump that is exclusively expressed in neurons. ${ }^{2,3}$ The hallmark of $\mathrm{AHC}$ is recurrent 
Table 1. Common causes of apnoea in term infants

\begin{tabular}{|l|l|}
\hline Causes of apnoea in term infants \\
\hline Central nervous system & Perinatal asphyxia \\
\hline Intracranial haemorrhage \\
\hline Infection & Sepsis \\
& Meningitis or encephalitis \\
\hline Respiratory system & Pneumothorax \\
\hline Pneumonia \\
\hline Respiratory distress syndrome \\
\hline Metabolic & Upper airway obstruction \\
\hline Hypoglycaemia \\
\hline Hyponatremia \\
\hline Inborn errors of metabolism \\
\hline Severe anaemia \\
\hline Cardiovascular system & Polycythaemia \\
\hline Cyanotic congenital heart disease \\
\hline Congestive heart failure
\end{tabular}

episodes of hemiplegia alternating between either side of the body. Paroxysmal dystonia, oculomotor abnormalities and dysautonomic phenomena often occur alone or with hemiplegic attacks, and may be triggered by emotional change, startling to bright lights or sound, exertion or specific foods. ${ }^{4}$

Three mutations-p.Glu815Lys, p.Asp801Asn and p.Gly947Arg - account for about $60 \%$ of cases with AHC. ${ }^{5}$ The p.Glu815Lys mutation carries a severe phenotype with neonatal onset of paroxysmal events, drug-resistant epilepsy, and poor neurodevelopmental outcome. Paciorkowski et al. reported a child with a novel heterozygous ATP1A3 mutation (p.Ile363Asn) with neonatal onset of life-threatening prolonged apnoea necessitating tracheostomy, prolonged ventilator support and eventually had severely impaired developmental outcome. $^{6}$

The de novo variant (NM_001256214.2(ATP1A3): c. $2960+2$ delT) in our case is not present in population databases (Genome Aggregation Database ${ }^{7}$ ) and has not been reported before. The sequence variant occurs at intron 21 of the ATP1A3 gene. It does not directly change the encoded amino acid sequence of the ATP1mA3 protein, but affects the consensus splice site of the intron, and hence was predicted to alter the length of the protein. We hypothesise this would affect the function or the stability of the enzyme i.e. a hypomorphic allele, which is consistent with the disease mechanism. ${ }^{8}$ Based on current evidence, this variant has been classified as likely pathogenic based on ACMG/AMP guidelines for interpretation of sequence variants.

There is no specific therapy for paroxysmal events in AHC although flunarizine, a calcium channel blocker, and topiramate, an antiepileptic medication, are more widely used. In a study of 30 patients, flunarizine reduced the duration and frequency of attacks in $50 \%$ of patients and decreased intensity in $32 \% .{ }^{9}$ Buccal midazolam, rectal diazepam and chloral hydrate are useful to abort attacks by inducing sleep. ${ }^{10}$ Intranasal midazolam, which is used in our case, is generally safe in neonates given its quick onset and short duration of action.

In summary, we describe a term neonate with recurrent apnoea beginning in the first 24 hours of life. After exclusion and treatment for common causes of apnoea, she was found to have alternating hemiplegia of childhood. A clinical exome study for term infants with treatment refractory and cryptogenic apnoea may help unveil a unifying diagnosis and guide management.

\section{Acknowledgement}

The authors would like to thank the parents of the infant described for sharing her details.

\section{REFERENCES}

1. Poblano A, Marquez A, Hernandez G. Apnea in infants. Indian J Pediatr 2006;73:1085-8.

2. Sasaki M, Ishii A, Saito Y, et al. Genotype-phenotype correlations in alternating hemiplegia of childhood. Neurology 2014;82:482-90.

3. Holm R, Toustrup-Jensen MS, Einholm AP, et al. Neurological disease mutations of alpha3 $\mathrm{Na}+, \mathrm{K}+$-ATPase: Structural and functional perspectives and rescue of compromised function. Biochim Biophys Acta 2016;1857:1807-28.

4. Rosewich H, Sweney MT, DeBrosse S, et al. Research conference summary from the 2014 international task force on ATP1A3-related disorders. Neurol Genet 2017;3:e139.

5. Capuano A, Garone G, Tiralongo G, et al. Alternating Hemiplegia of Childhood: Understanding the Genotype-Phenotype Relationship of ATP1A3 Variations The Application of Clinical Genetics 2020;13:71-81.

6. Paciorkowski AR, McDaniel SS, Jansen LA, et al. Novel mutations in ATP1A3 associated with catastrophic early life epilepsy, episodic prolonged apnea, and postnatal microcephaly. Epilepsia 2015; 56:422-30. 
7. Karczewski KJ, Francioli LC, Tiao G, et al. The mutational constraint spectrum quantified from variation in 141,456 humans. Nature 2020;581:434-43.

8. Rosewich H, Thiele H, Ohlenbusch A, et al. Heterozygous de-novo mutations in ATP1A3 in patients with alternating hemiplegia of childhood: a whole-exome sequencing gene-identification study. Lancet Neurol 2012;11:764-73.

9. Pisciotta L, Gherzi M, Stagnaro M, et al. Alternating Hemiplegia of Childhood: Pharmacological treatment of 30 Italian patients. Brain Dev 2017;39:521-8.

10. Samanta D. Management of alternating hemiplegia of childhood: A review. Pediatr Neurol 2019;103:12-20.

Natalie Yi Ting Koh, ${ }^{1} M B B C h B A O($ (reland), Jocelyn Yi Xiu Lim, ${ }^{2}$ MRCPCH (UK), Sylvia Kam, ${ }^{3}$ MGenCouns,
Nirmal Kavalloor Visruthan, ${ }^{4}{ }_{M R C P C H}(U K)$,

Ai Ling Koh, ${ }^{3}$ MRCPCH (UK), Jan Hau Lee, ${ }^{5}{ }_{\text {MRCPCH (UK), }}$ Terrence Thomas, ${ }^{2}$ MRCPCH (UK)

${ }^{1}$ Department of Paediatrics, KK Women's and Children's Hospital, Singapore ${ }^{2}$ Neurology Service, Department of Paediatrics, KK Women's and Children's Hospital, Singapore

${ }^{3}$ Genetics Service, Department of Paediatrics, KK Women's and Children's Hospital, Singapore

${ }^{4}$ Department of Neonatology, KK Women's and Children's Hospital, Singapore ${ }^{5}$ Children's Intensive Care Unit, KK Women's and Children's Hospital, Singapore

Correspondence: Dr Jocelyn Lim, Neurology Service, Department of Paediatrics, KK Women's and Children's Hospital, 100 Bukit Timah Road, Singapore 229899.

Email: jocelyn.lim.y.x@singhealth.com.sg 